\title{
Measuring anti-convulsant levels in the accident and emergency department
}

\author{
R. McGLONE AND P. PRITTY
}

Department of Accident and Emergency Medicine, Derbyshire Royal Infirmary, Derby, England

\section{SUMMARY}

A prospective study involving 57 epileptic adults who presented to the Derbyshire Royal Infirmary Accident and Emergency Department with fits has shown that 57\% were in the sub theraputic ranges.

\section{METHOD}

A prospective study involving 57 epileptics who presented to the accident and emergency department following a fit was carried out. A questionnaire was completed by the doctor on duty and each patient had blood taken for estimation of anticonvulsant levels.

\section{RESULTS}

\section{Anti-convulsant levels}

Forty-one out of 57 known epileptics had their anti-convulsant levels estimated, some of these on multiple therapy (Table 1).

The reasons for levels not being taken were that four were currently not on treatment, three had levels done recently, two were on benzodioazepines, two were a late presentation and one had gastro-enteritis and had not been taking tablets; for four, no reason was given.

Fifty-seven per cent of anti-convulsant levels in the study were in the sub-therapeutic

Correspondence: $\operatorname{Dr}$ R. McGlone, Registrar in Accident and Emergency Medicine, Derbyshire Royal Infirmary, Derby DE1 2QY, England 
Table 1 Anti-convulsant levels ( $\mu \mathrm{mol} / \mathrm{litre}$ ) of 57 known epileptics*

\begin{tabular}{|c|c|c|}
\hline Phenytoin & Phenobarbitone & Carbamazepine \\
\hline 44 & 88 & 13 \\
\hline 45 & 52 & 17 \\
\hline 70 & 28 & $<10$ \\
\hline 18 & 23 & $<10$ \\
\hline 20 & 22 & 17 \\
\hline$<10$ & 52 & $* 53$ \\
\hline 12 & $* 318$ & 23 \\
\hline 28 & 66 & 33 \\
\hline 53 & 41 & 22 \\
\hline 55 & 35 & 14 \\
\hline 44 & ${ }^{*} 175$ & 13 \\
\hline$<10$ & 17 & 24 \\
\hline$<10$ & $<10$ & \\
\hline$<10$ & $<10$ & \\
\hline$<10$ & $<10$ & \\
\hline$<10$ & 93 & \\
\hline 49 & 20 & \\
\hline$<10$ & 164 & \\
\hline \multicolumn{3}{|l|}{54} \\
\hline \multicolumn{3}{|l|}{37} \\
\hline \multicolumn{3}{|l|}{$<10$} \\
\hline \multicolumn{3}{|l|}{12} \\
\hline 42 & & \\
\hline
\end{tabular}

*Therapeutic range ( $\mu \mathrm{mol} / \mathrm{litre})$ : carbamazepine, $16-40$; phenytoin, 40 100; phenobarbitone, 65-70 (figures below therapeutic range in italics, figures above with asterisks).

range and $25 \%$ of the levels were below the sensitivity of the asay. Only two of the 20 cases in the therapeutic range were in the upper half of the range.

Seventeen patients were on combination therapy. In five patients, levels of both drugs were below the therapeutic range and a further five had subtherapeutic levels of one drug.

\section{DISCUSSION}

Many of these patients had not had their anti-convulsant levels taken for some time. However, this certainly did not indicate neglect of their condition. Once the correct $\stackrel{\sim}{\mathscr{N}}$ dose has been found there is little merit in checking levels unless problems arise.

Compliance is a major problem: $40 \%$ of out-patients do not take all the medication prescribed (Richens, 1978). The optimal time to check levels immediately after a fit and not in the out-patient department.

Phenytoin, phenobarbitone and ethosuximide have plasma half-lives which are sufficiently long for the timing of the sample not to be critical. Carbamazepine has a ? short half-life and the sample should ideally be between 4 and 6 hours after the last dose. 
The value of monitoring valproate levels is uncertain. It has a short half-life and there does not seem to be a direct relationship between plasma concentration and clinical control of epilepsy. The survey predictably showed a large number of patients with subtherapeutic levels. Toxic levels are rare and the only patient who had a significant toxic level had, in fact, taken a deliberate overdose.

Some patients have fits even when their drug levels are within the theraputic range. Their level of medication is clearly inadequate and their doctor needs to know this. However, many patients are fitting because they are not taking their medication as prescribed. Estimating the drug level at the time of presentation to an accident and emergency department has revealed a higher rate of non-compliance than in an outpatient department and it should become routine.

\section{CONCLUSION}

As compliance is a major problem it is important to discover whether the patient is indeed taking his tablets and the optimal time to do this is immediately following a fit. Levels taken in out-patients may not necessarily reflect a true picture and may lead to an inappropriate increase in dosage.

\section{REFERENCE}

Richens A. (1978) Choice of drugs in epilepsy. Prescribers fournal 18, 125-33.

Received 28 April 1985; accepted 8 November 1985 